\title{
SOCIAL INTERACTIONS WITH FAMILIES
}

\author{
E. Leshtarova* \\ Department of Social Activities, Faculty of Medicine, Trakia University, Stara Zagora, Bulgaria
}

\begin{abstract}
The subject of this article is the social interactions between teachers and parents in kindergarten in order to build partnership to improve their social competencies. A three - year programme for enrichment of parents' knowledge of children's development, improvement of communication skills, self-analysis of parents and teachers in the process of interaction with children is presented. Some conclusions are made based on this successfully built teachers-parents community, expanding the knowledge and experience of each member, improving the communication skills and developing tolerance and acceptance of differences, shaping the expected behavior of children.
\end{abstract}

Key words: social interactions, family, teachers, programme, training

\section{INTRODUCTION}

Interaction between kindergarten and family acting as social and educational institutions can be regarded as social-pedagogical establishment. It reflects the process of mutual influence, enrichment and development and forms positive or negative attitudes and the resulting behavioral tactics.

The most important features of this interaction are productive interactions between all the participants, their shared responsibility and influence forming a unity.

Society is also interested in this interaction, as it realizes its functions to adolescents by family and kindergarten. Through the implementation of specific educational potential of both institutions, the common tasks and main purpose are solved.

As a whole, the types of teacher-parent interactions applied in the pedagogical theory and practice continue to be meaningful:

Mutual knowledge of the two groups bears the specifics of the roles carried out by each of them. The peculiarities of these roles are not completely realized. The idiosyncrasy of children's individuality and its reshaping by the kindergarten reality and requirements are even less updated according to modern lifestyle, children's age and the characteristics of each task.

\footnotetext{
*Correspondence to: Emilia Leshtarova, Trakia University, Faculty of Medicine, Department of Social Activities, 11 "Armeiska" street, 6000, Stara Zagora, Bulgaria
}

Unification of the main aim and methods of achieving it

It includes:

- content and objectives of educational process in the kindergarten;

- requirements of parents and teachers with respect to social development;

- parents and teachers' requirements regarding a successful life and personal fulfillment social and emotional skills, attitudes and competencies which are socially significant for the child;

- activities between families and kindergarten related to the integrity of child development - successes, problems, corrective action, etc.;

- showing positive attitude to the child, acceptance and affection stimulate humane thinking and proper behaviour.

\section{DISCUSSION}

The modern vision of the role and importance of family upbringing conveys the message that the family is the center of the ecological system, which makes the family a kind of a filter between the child and the outside world and kindergarten.

The initial practical behavior models that children follow in the community are initially formed in the family. Organizing and promoting interaction between kindergarten and family is crucial because family relationships are part of society and are influenced by socio-economic changes. Each child comes from an environment that has its own atmosphere. This inevitably puts imprint 
on each family member's attitude to the child and resonates on all those with whom they come in contact. The family is an important educational institute, and the mother and father are the first teachers of their children. Parents influence the development of children's personality with their knowledge, a system of values and behavior patterns. The parental love and the emotional bond between parents and children are of great importance. The family atmosphere favors to its utmost intellectual and spiritual enrichment of the individual, contribute to the development of self-knowledge and self-perception of the child.

Considering parents as our partners modern European education strategy raises the issue of transition from social commitment to the democratic participation of the family in education.

The realization of pedagogical competences of people employed in the education system in the context of the interaction "educational institutions-family" requires new communication strategies for cooperation with parents whose conceptual justification involves defining the specifics of the factors of socioeducational system. Teacher-parent-child cooperation is essential for maintaining stable relationship in the kindergarten.

Some information about the structure and content of family relationships and the status of the child in it; family atmosphere and emotional well-being of the child in it; particularities of family culture, ethnicity and occupation of parents; dominant type of education; level of knowledge, skills and interests of children and parents is needed In order to optimize the interaction between family and kindergarten.

All this requires the need to seek new ways for permanent and effective contact not only with the parents but also with the relatives and other people who are part of the child's environment. "Pedagogical education of parents" means systematically pursued and theoretically based programme, which aims to transfer knowledge and form corresponding ideas and habits of participants in various areas of family upbringing.

The success of the educational programme largely depends on the parents' involvement in kindergarten life based on partnership and cooperation. That means parents will be direct participants in kindergarten's reality, they will transform from viewers to teacher's assistants and become a source of ideas, knowledge, experience.

We offer a programme containing active forms of interaction through which family members can build on their parental competencies.

We are convinced that adults learn best through active participation in a well-planned process that reflects their interests and contribute to improving their ability to communicate and interact with children. On the other hand, the programme involves individualized interaction, attitude and training, ensures compliance with personal needs of family members and teachers. It relies on understanding, learning and upgrading through experience - personal and professional to provide the best support for each child.

Both parents and teachers benefit from influencing and completing each other in the performance of their social roles and functions. Development of the following approaches was used in this programme: humane - personal, individual and active.

\section{MATERIALS AND METHODS}

\section{A three-year programme}

Aim: To stimulate social competences of teachers and parents.

Tasks:

1. Expanding the knowledge of parents about the specifics of preschool age - physical, social, emotional, cognitive development of children's personality.

2. Improving communication skills and interaction between parents and children.

3. Reflection - introspection, self-esteem of parents and teachers in the process of interaction with children.

The target groups are teachers and parents.

Activities:

1. "Do we know the child? - Meeting in Blitz group;

2. "To love and accept the child" - Training;

3. "How to show approval or disapproval of child behavior" - Training;

4. "How to stimulate the autonomy of the child?" - Training;

5. "Solving problems and conflicts in the family" - Training;

6. "How to show tenderness and emotional support to the child?" - Training;

7. "How to talk so your child listens and how to listen so that the child speaks" - Training;

8. "The child artist" - Training;

9. "Children negativism, stubbornness and whims" - Training; 
10. "How to stimulate the child's interest in learning" - Training.

The three-year programme can be implemented as follows: 1-4 themes in the second age group; 5-7 themes in the pre-school group 5 years old; 8-10 themes in the preschool group - 6 years old.

With this study we aim to develop social competences of teachers and parents.

The aim of the study was carried out by solving tasks through systematic and consistent deployment of group interaction between teachers and parents.

The results give us reason to believe that the target was achieved.

\section{RESULTS}

The theoretical analysis on the problem of the relationship of children to family members entitles us to say that:

- Communication with parents is vital for children for their full mental and social development.

- In social psychological terms different patterns of communication in the family as a social community are offered: adultchild; child - adult.

- The family balance, fairness, reasonable requirements build mental and psychological balance in children.

\section{CONCLUSION}

The results allow us to make the following conclusions:

- The established teacher - parent community gives a sense of belonging;

- Theoretically and practically, they learn from each other, expanding the knowledge and experience of all members;

- Working together builds trust between community members;

- The members of the community built selfcorrecting skills;

- Speaking and listening skills improvement;

- Developing a sense of sympathy through first-hand experience;

- Developing tolerance and acceptance of differences;

- Modeling the target behavior in children;

- Participation in an open and meaningful exchange of ideas related to the child's personality development. 